\title{
NONLINEAR WAVEPACKETS IN PAIR-ION AND ELECTRON-POSITRON-ION PLASMAS
}

\author{
I. KOURAKIS \\ Centre for Plasma Physics, Department of Physics and Astronomy, \\ Queen's University Belfast, BT7 1 NN Northern Ireland, UK \\ E-mail: i.kourakis@qub.ac.uk,www.kourakis.eu \\ R. ESFANDYARI \\ Azarbaijan University of Tarbiat Moallem, Faculty of Science, \\ Department of Physics, 51745-406, Tabriz, Iran \\ P. K. SHUKLA \\ Institut für Theoretische Physik IV, Fakultät für Physik und Astronomie, \\ Ruhr-Universität Bochum, D-44780 Bochum, Germany

\section{F. VERHEEST} \\ Universiteit Gent, Sterrenkundig Observatorium, Krijgslaan 281, \\ B-9000 Gent, Belgium \\ and \\ School of Physics, Howard College Campus, University of KwaZulu-Natal, \\ Durban 4041, South Africa

\section{N. F. CRAMER} \\ School of Physics, University of Sydney, New South Wales 2006, Australia \\ The occurrence of amplitude-modulated electrostatic and electromagnetic \\ wavepackets in pair plasmas is investigated. A static additional charged back- \\ ground species is considered, accounting for dust defects or for heavy ion \\ presence in the background. Relying on a two-fluid description, a nonlinear \\ Schrödinger type evolution equation is obtained and analyzed, in terms of the \\ slow dynamics of the wave amplitude. Exact envelope excitations are obtained, \\ modelling envelope pulses or holes, and their characteristics are discussed.
}

\section{Introduction}

In their widespread textbook picture, charge-neutral electron-ion $(e-i)$ plasmas are modelled as large ensembles of electrons $e^{-}$(charge $q_{e}=-e$, mass 
$\left.m_{e}\right)$ and positive ions $i^{+}$(charge $q_{i}=+Z_{i} e$, mass $m_{i} \gg m_{e}$ ). The small ion-to-electron mass ratio is associated with $e-i$ plasma features, which are most often implicitly taken for granted: for instance, the electron and ion plasma frequency $\omega_{p, s}=\left(4 \pi n_{s} q_{s}^{2} / m_{s}\right)^{1 / 2}$ (for species $s$; here, $s=e$ or i) and cyclotron frequency $\omega_{c, s}=q_{s} B / m_{s} c$ are clearly different, thus allowing for a clear distinction among corresponding time and (e.g., Debye) length) scales and associated wave phenomena. ${ }^{1}$ Pair plasmas (p.p.) are distinct from this picture, in that they consist of two ion populations (say, $1^{+}$and $2^{-}$) of equal mass and opposite charge (i.e., $q_{1}=-q_{2}=+Z e$ and $\left.m_{1}=m_{2}=m\right)$. The pair ion densities at equilibrium, although equal in a symmetric ("pure") p.p. configuration, may differ if the charge balance is affected by a 3 rd population, e.g. a massive charged defect species $3^{ \pm}$ (e.g. dust $^{2}$ ), assumed present as a stationary background. No plasma or cyclotron frequency separation occurs in $p . p$.; furthermore, a variety of novel physical phenomena (e.g. absence of Faraday rotation) characterizes electrostatic (ES) and electromagnetic (EM) wave propagation in such plasmas. ${ }^{3}$ Although this simple description of pair plasmas was originally introduced to model (for $Z=1$, here) electron-positron (e-p) plasmas ${ }^{\text {a }}$ (yet overseeing $e-p$ annihilation-recombination processes, here neglected throughout), it may claim to provide a consistent model of fullerene-ion pair plasma configurations which were recently successfully produced in experiments. ${ }^{4}$ Significant research effort has recently focused on the properties of linear and nonlinear wave propagation in such plasmas.

Plasma wave observations, both in Space and in the laboratory, provide abundant evidence for the existence of spatially localized propagating wave structures, e.g. in the form of a localized envelope pulse confining (modulating) a fast carrier wave. ${ }^{5}$ Modulated wavepackets of this form may occur as a result of modulation instability (MI), when nonlinearity at the first stages of the amplitude instability is balanced by group dispersion. This physical mechanism is reminiscent of energy localization phenomena in nonlinear optics, hydrodynamics and biophysics. ${ }^{6}$ As regards pair plasmas, nonlinear modulated wavepackets may occur either as $\mathrm{ES}^{7,8}$ or electromagnetic $\mathrm{EM}^{9}$ wavepackets.

The aim of this brief report is to review existing results on linear waves and nonlinear envelope structures propagating in pair plasmas. A two-fluid plasma model is introduced for this purpose, for the pair species; a third massive component is taken to be stationary, referring to three-component

a The 3rd species type then represents ions, in electron-positron-ion (e-p-i) plasmas. 
pair plasmas of, say, either the type $i^{+} i^{-} 3^{ \pm}$(i.e., p.p. "doped" with dust defects) or $e^{-} p^{+} i^{+}$. No assumption is made on the density ratio $n_{+} / n_{-}$or the temperature ratio $T_{+} / T_{-}$, since one of our intentions is to point out the role of a possible pair-component asymmetry in the plasma configuration (also, of the fixed background species $3^{ \pm}$) on the properties of nonlinear ES waves. High frequency ES waves in $e-p-i$ plasmas are also included in this picture.

\section{A two-fluid model for ES wavepackets in pair plasmas}

Consider a two-plasma-fluid model, described by the dynamical equations

$$
\begin{gathered}
\frac{\partial n_{j}}{\partial t}+\nabla \cdot\left(n_{j} \mathbf{u}_{j}\right)=0 \\
\frac{\partial \mathbf{u}_{j}}{\partial t}+\mathbf{u}_{j} \cdot \nabla \mathbf{u}_{j}=-s_{j} \frac{Z e}{m} \nabla \phi-\frac{1}{m n_{j}} \nabla p_{j},
\end{gathered}
$$

for the density $n_{j}$ and the velocity $\mathbf{u}_{j}$ of the $j$-th particle species $(j=$ $\left.1^{+}, 2^{-} \equiv+,-\right)$. The equation of state $p_{j} \sim n_{j}^{\gamma}$ is assumed for the pressure $p_{j}(\gamma=1+2 / f$ is the specific heat ratio, for $f$ degrees of freedom), while $p_{j, 0}=n_{j, 0} k_{B} T_{j}$ is assumed at equilibrium (the Boltzmann constant $k_{B}$ preceding the temperature $T_{j}$ will be omitted where obvious). The difference in charge sign is expressed by $s_{j}=q_{j} /\left|q_{j}\right|= \pm 1$. The system is closed by Poisson's equation

$$
\nabla^{2} \Phi=-4 \pi \sum_{s} q_{s} n_{s}=4 \pi e\left(Z n_{-}-Z n_{+}-s_{3} Z_{3} n_{3}\right)
$$

Note that $s_{3}= \pm 1$ may account for either positive or negative background ions $^{\mathrm{b}}$. The charge balance, as expressed via the neutrality hypothesis (at equilibrium): $Z n_{+, 0}-Z n_{-, 0}+s_{3} Z_{3} n_{3}=0$, is obviously affected by the presence of the $3 \mathrm{rd}$ background species (of density $n_{3}=\mathrm{cst}$.). The case of "pure" pair (two-component, e.g. $e-p$ ) plasmas is thus recovered for $n_{3}=0$ (i.e. $n_{+, 0}=n_{-, 0}$ ), while $n_{3} \neq 0$ in general (in $e^{-} p^{+} i^{+}$or, say, $X^{+} X^{-} d^{ \pm}$ type plasmas). A one-dimensional geometry will be adopted here (hence $f=1$ and $\nabla=\partial / \partial x$ ), although a (richer) multi-dimensional description may also be found in the bibliography. ${ }^{8}$

In the following, reduced expressions will be implicitly assumed for all quantities, scaling space $x$ and time $t$ units are the Debye length $\lambda_{D,-}=c_{s} / \omega_{p,-}$ and the inverse plasma frequency $\omega_{p,-}^{-1}$, respectively; also,

\footnotetext{
bi.e., for dust $d^{ \pm}$(viz. $s_{3}= \pm 1$ ) in "doped p.p.", or for ions $\left(s_{3}=+1\right)$ in $e-p$ - $i$ plasmas.
} 
the density, velocity and electric potential variables are scaled by $n_{-, 0}$, $c_{s}=k_{B} T_{-} / m$ and $k_{B} T_{-} / Z e$, respectively.

\section{Linear electrostatic wave dispersion properties}

The linear (small amplitude) approach consists in assuming perturbations of all elements, say $S_{l}$, of the state vector $\mathbf{S}=\left(\left\{n_{j}, u_{j} ;\right\} ; \phi\right)$ (here $l=$ $1, \ldots, 5 ; j=1,2)$ near equilibrium $\mathbf{S}_{0}=\left(\left\{n_{j, 0}, 0 ;\right\} ; 0\right)$, in the form $\epsilon S_{j} \approx$ $S_{1, l}^{(1)} \exp i(k x-\omega t)$; here $S_{1, l}^{(1)}$ is the wave amplitude (assumed constant for a while), while $k$ and $\omega$ denote(s) the carrier wavelength and frequency, respectively. Inserting into Eqs. (1)-(3), one obtains the dispersion relation:

$$
\frac{1}{\omega^{2}-3 k^{2}}+\frac{\beta}{\omega^{2}-3 \sigma \beta^{2} k^{2}}=1,
$$

where $\beta=n_{+, 0} / n_{-, 0}$ is the density ratio ${ }^{\mathrm{c}}$ and $\sigma=T_{+} / T_{-}$is the temperature ratio, among the pair components. A bi-quadratic polynomial equation is thus obtained and is straightforward to solve, providing two branches, say $\omega=\omega_{L}(k)$ and $\omega=\omega_{U}(k)$. The exact expressions are presented and analyzed in Ref. ${ }^{7}$ The lower branch $\omega_{L}$ describes an acoustic mode, as $\omega_{L}(0)=0$, while the upper one bears a Langmuir-type form, featuring a cutoff frequency $\omega_{U}(0)=(1+\beta)^{1 / 2}$ (in units of $\omega_{p,-}$; see above). Interestingly, no acoustic mode in principle exists for perfectly symmetric p.p. configurations; to see this, set $\beta=\sigma=1$ in $(4)^{\mathrm{d}}$, to obtain $\omega^{2}=2+3 k^{2}$ $\left(\right.$ cf. literature $\left.{ }^{4}\right)$. Asymmetric p.p. are henceforth implicitly assumed everywhere. Considering the behavior of both branches near $k=0$, we obtain

$$
\omega_{L}(k) \approx c_{s}^{2} k^{2}, \quad \omega_{U}(k) \approx\left(\omega_{c}^{2}+c_{s}^{2} k^{2}\right)^{1 / 2} .
$$

We note that the cutoff frequency $\omega_{c}=(1+\beta)^{1 / 2}$ depends on the background (third) species' concentration (via $\beta$ ), while the sound speed $c_{s}=[3 \beta(1+\sigma \beta) /(1+\beta)]^{1 / 2}$ also depends on the pair component temperature asymmetry via $\sigma$. This behavior is depicted in Fig. 1. The dispersion reported in fullerene experiments ${ }^{4}$ is recovered for $\beta=\sigma=1$, as expected.

The amplitudes of the linear oscillations are obtained in terms of the electric potential perturbation $\phi_{1}^{(1)}=\psi$ as

$$
n_{+, 1}^{(1)}=\frac{\beta k^{2}}{\omega^{2}-3 \sigma \beta^{2} k^{2}} \psi=\frac{\beta k}{\omega} u_{+, 1}^{(1)}, \quad n_{-, 1}^{(1)}=-\frac{k^{2}}{\omega^{2}-3 k^{2}} \psi=\frac{k}{\omega} u_{-, 1}^{(1)} .
$$

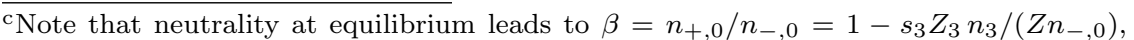
so a value above (below) unity implies a negatively (positively) charged third species presence in the background, viz. $s_{3}=-1(+1)$. Obviously, $\beta=1$ in "pure" p.p.

${ }^{\mathrm{d}}$ This seems to suggest an asymmetry among the pair ion species in the experiment of Oohara and Hatakeyama ${ }^{4}$ where the observation of an acoustic mode was reported.
} 


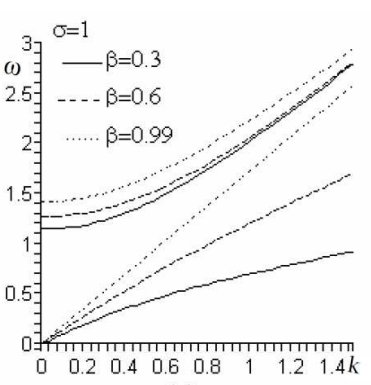

(a)

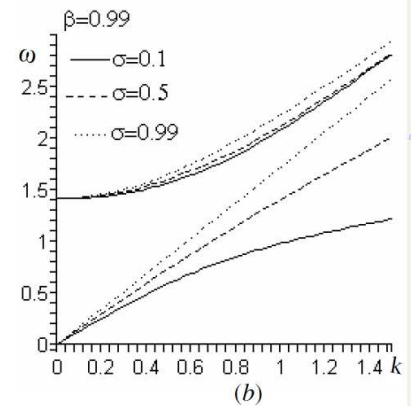

(b)

Fig. 1. The two dispersion curves defined by Eq. (4) are depicted: frequency $\omega$ vs. wavenumber $k$ (reduced quantities; see in the text). After Ref. 7.

The subscripts +/- will be used, where obvious, to distinguish the positive/negative ions (or, positrons/electrons). These expressions may be used in plasma diagnostics, to trace the presence of charged defects in the background and/or pair-ion asymmetry in real (experimentally produced) p.p.

\section{Multiple scales theory for modulated ES wavepackets}

We shall now consider a small (yet finite) deviation from the equilibrium state $\mathbf{S}_{\mathbf{0}}$, by allowing for a weak time and space dependence (modulation) of the wave's amplitude $A_{j}$. All state variables $S_{l}(l=1, \ldots, 5)$ are assumed to vary as $S_{l}(x, t)=S_{0, l}+\sum_{m=1}^{\infty} \epsilon^{n} \sum_{L=-\infty}^{\infty} S_{L, l}^{(n)}(\xi, \tau) \exp [i L(k x-\omega t)]$ where $\epsilon \ll 1$ is a (real) smallness parameter (the equilibrium state vector $\mathbf{S}_{\mathbf{0}}$ was defined above). Here, the superscript $n$ (subscript $L$, respectively) denote(s) the expansion order in $\epsilon$ (the phase harmonic order $m$ ). The reality condition $S_{L, l}^{(n)}=\left(S_{-L, l}^{(n)}\right)^{*}$ is implied; the star superscript denotes the complex conjugate. The wave amplitudes $S_{L, l}^{(m)}$ depend on the stretched ("slow") coordinates $\xi=\epsilon(x-\lambda t)$ and $\tau=\epsilon^{2} t$, where $\lambda=v_{g}=d \omega / d k$ is the wave group (envelope propagation) velocity. We proceed by substituting into Eqs. (1)-(3) and isolating various orders (in $\left.\epsilon^{n}\right)$, for the $L$-th harmonic contributions; details (omitted here for brevity) can be found elsewhere. ${ }^{5}$

The algebra is tedious yet straightforward. The equations for $n=1$ reproduce the solution and dispersion characteristics presented in $\S 3$. The equations for $n=2$ produce the (amplitudes of the) 2nd order harmonics, as well as a zeroth order (direct current) term. The solution thus obtained,

$$
\phi \approx \epsilon \psi \cos \theta_{c}+\epsilon^{2}\left[\phi_{0}^{(2)}+\phi_{1}^{(2)} \cos \theta_{c}+\phi_{2}^{(2)} \cos 2 \theta_{c}\right]+\mathcal{O}\left(\epsilon^{3}\right),
$$


e.g., for the electric potential $\phi$, represents a carrier wave (fast phase $\theta_{c}=$ $k x-\omega t$ ); a set of similar expressions are obtained for $n_{+/-}$and $\mathbf{u}_{+/-}$. At this stage, one is after an evolution equation for the potential correction $\psi$; once this is solved, anticipating a (complex) solution in the form $\psi=\psi_{0} \exp i \Theta$, the first-order corrections to all quantities directly follow from (6) above. The real variables $\psi_{0}$ and $\Theta$ physically represent the potential (wavepacket) real (i.e., measurable) amplitude and a (small) phase correction, leading to a weakly varying total phase $\theta=\theta_{c}+\epsilon^{2} \Theta+\mathcal{O}\left(\epsilon^{3}\right)$.

A nonlinear Schrödinger-type equation (NLSE) arises, at order $n=3$, as a compatibility equation (ensuring secular term annihilation). It reads

$$
i \frac{\partial \psi}{\partial \tau}+P \frac{\partial^{2} \psi}{\partial \xi^{2}}+Q|\psi|^{2} \psi=0 .
$$

The dispersion coefficient $P$ is related to the dispersion characteristics as $P=d^{2} \omega / 2 d k^{2}$, as obtained from (4) above (for each branch, separately). The lengthy expression for the nonlinearity coefficient $Q=Q(k ; \beta, \sigma)$ (here omitted, for brevity) is given by Eq. (19) in Ref. 7. Different expressions are obviously obtained for waves obeying the upper and lower dispersion curves (see in $\S 3$ above).

\section{Modulational instability \& localized envelope excitations}

The evolution of the electric potential amplitude $\psi$ depends on the coefficients $P$ and $Q,{ }^{5}$ whose analytical behavior is straightforward to investigate in terms of the physical parameters involved. The key element in this analysis, as discussed below, turns out to be the quantity $P / Q$ : its sign $(+/-)$ determines the regions where harmonic oscillations are unstable/stable, as well as the generic type (bright/dark, respectively, to be explained in the following) of envelope excitations, while its magnitude tunes their characteristics (amplitude $\psi_{0}$, width $L$ ) via a relation in the form $L \sim \psi_{0}(P / Q)^{1 / 2}$.

Modulational instability. Summarizing all physically relevant information - yet omitting details ${ }^{5}$ - one may consider a harmonic solution of (8) in the form $\psi=\psi_{0} \exp \left(i Q\left|\psi_{o}\right|^{2} \tau\right)$; the standard (linear) stability analysis then leads to the dispersion relation: $\tilde{\omega}^{2}=P \tilde{k}^{2}\left(P \tilde{k}^{2}-2 Q\left|\tilde{\psi}_{0}\right|^{2}\right)$, where $\tilde{\omega}, \tilde{k}$ and $\tilde{\psi}_{0}$ denote the frequency, wavenumber and amplitude perturbation(s), respectively. One sees that, for $P Q>0$, a critical wavenumber threshold $\tilde{k}_{c r}=\left|\tilde{\psi}_{0}\right|(2 Q / P)^{1 / 2}$ exists, below/above which, the envelope is unstable/stable (i.e., for perturbation wavelengths longer/shorter than $\left.2 \pi / k_{c}\right)$; the maximum instability growth rate $|\operatorname{Im} \tilde{\omega}|_{\max }=Q\left|\tilde{\psi}_{0}\right|^{2}$ occurs at $k_{m}=k_{c} / \sqrt{2}$. If $P Q<0$, the amplitude will be stable to perturbations. This 
is essentially the Benjamin-Feir instability mechanism in hydrodynamics. ${ }^{6}$
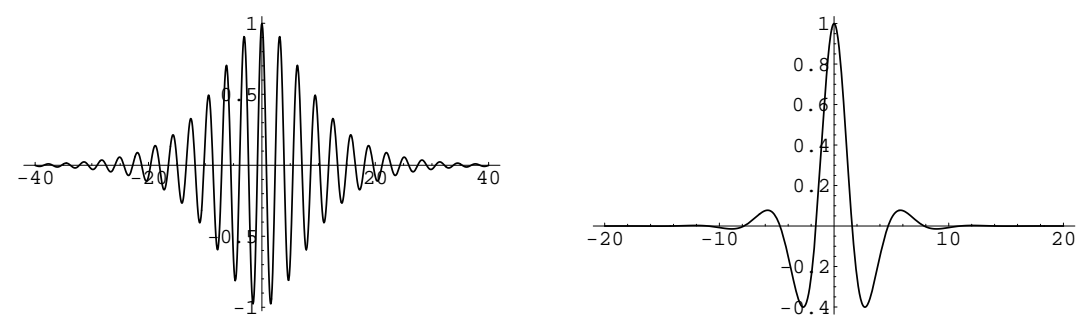

Fig. 2. Bright type modulated wavepackets (for $P Q>0$ ), for two different (arbitrary) sets of parameter values. After Ref. 5 .

Envelope excitations. The NLSE (8) defines an integrable dynamical system, which possesses various types of stationary profile spatially localized solutions, in the form of envelope solitons; the analytical form of the latter can be rigorously obtained via the Inverse Scattering Transform (IST) method. The remarkable properties of solitons (e.g. longevity and robustness against perturbations, shape-invariance through collisions), enumerating which goes far beyond our scope here (refer to specialized literature ${ }^{6}$ ) makes these solutions a loyal working horse for ES wavepacket theories.
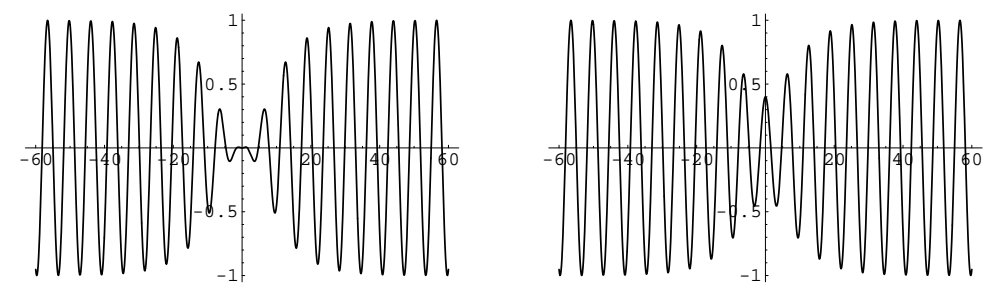

Fig. 3. Dark-type modulated wavepackets (for $P Q<0$ ) of the black (left) and grey (right) kind. See that the amplitude never reaches zero in the latter case. After Ref. 5.

The modulated (electrostatic potential) wave amplitude forms resulting from the above analysis may be obtained via an ansatz of the form $\psi(\xi, \tau)=$ $\rho(\xi, \tau) \exp [i \Phi(\xi, \tau)]$, where $\rho$ and $\Phi$ are analytical (real) functions, to be determined upon substitution into the NLS. A number of exact solutions are thus obtained. ${ }^{5,10}$ Omitting algebraic details ${ }^{5,10}$ the physically relevant information of importance to us here is summarized in the following.

For positive values of the coefficient product $P Q>0$ (or, of the ratio 
$P(Q)^{\mathrm{e}}$, the NLSE (8) possesses bright-type soliton solutions representing a propagating potential envelope pulse (vanishing at infinity), which encloses the fast carrier wave oscillation; see Fig. 2. This type of solution is reminiscent of signal pulses in optical fibres. ${ }^{6}$

For negative values of the coefficient product $P Q<0$, we find darktype soliton solutions, representing a propagating potential envelope void (i.e., a potential hole); these excitations bear a finite (constant) value at infinity; see Fig. 3. Localized envelope solitons of this kind exist in the form of black solitons, bearing a vanishing value in the center (see Fig. 3a), or grey solitons, bearing a finite potential value everywhere (see Fig. 3b).
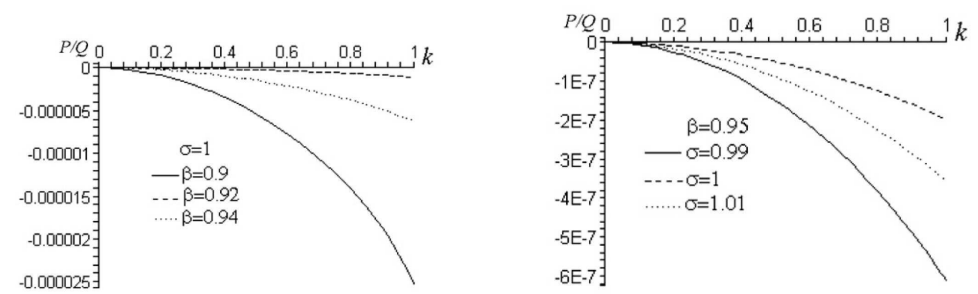

Fig. 4. The NLSE coefficient ratio P/Q corresponding to the lower (acoustic) dispersion branch $\omega_{L}$ is depicted against the (reduced) wavenumber $k$. (a) $\sigma=1$ and different values of $\beta$ are considered; (b) $\beta=0.95$, and $\sigma$ varies. Note that curves overlap. After Ref. 7 .

Pair plasmas - parametric investigation. As regards pair plasmas, the stability profile can be determined along the above guidelines. The analysis shows that the lower (acoustic) mode $\omega_{L}$ is stable (viz. $P / Q<0$; see Fig. 4) in all cases, for long wavelengths ${ }^{\mathrm{f}}$, while the upper (optic-type) branch $\omega_{U}$ is generally modulationally unstable (viz. $P / Q<0$; see Fig. 5). As a consequence, the lower mode favors the propagation of dark-type envelopes (Fig. 3), while bright envelopes (pulses; see Fig. 2) are expected to occur in the upper mode. Increasing $\beta$ (i.e. for $n_{+, 0}>n_{-, 0}$, implying a higher concentration of, say, negative background defects, or dust) leads to smaller (less extended) dark excitations (see Fig. 4a), while a positive background leads to the opposite effect. Temperature asymmetry (i.e. $\beta$ variation) is also seen to affect the characteristics of dark envelopes (see Fig. 4b). On

e The coefficient $Q$ is assumed not to vanish here. For vanishing $Q$, nonlinearity does not balance dispersion, so the analysis presented here fails. On the other hand, for vanishing $P$, one needs to resort to higher-order dispersion effects, here omitted.

${ }^{\mathrm{f}}$ Long wavelengths $\lambda=2 \pi / k$ (i.e. small wavenumbers $k$ ) are relevant with a fluid model (inevitably invalidated by overseen kinetic effects, e.g. Landau damping, for higher $k$ ). 
the other hand, bright envelopes, in the upper mode (though more likely to be experimentally observed than the acoustic mode; see comment in $\S 3$ ) bear no significant temperature asymmetry effect (notice the overlapping curves in Fig. 5).
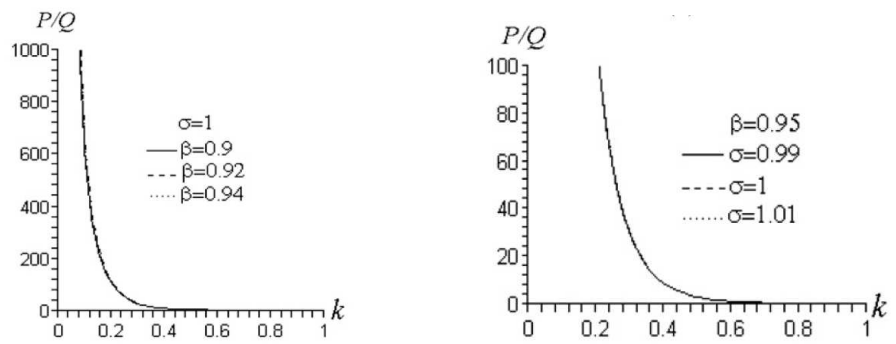

Fig. 5. JPA $4 \mathrm{~b}$ and $4 \mathrm{~d}$. The NLSE coefficient ratio $\mathrm{P} / \mathrm{Q}$ corresponding to the upper (optic) dispersion branch $\omega_{U}$ is depicted against the (reduced) wavenumber $k$. (a) $\sigma=1$ and different values of $\beta$ are considered; (b) $\beta=0.95$, and $\sigma$ varies. After Ref. 7 .

\section{A two-fluid model for EM wavepackets in pair plasmas}

We shall now consider electromagnetic excitations propagating in a magnetized pair plasma (in a uniform ambient magnetic field $\mathbf{B}_{\mathbf{0}}$ ). Retaining the above notation (unless otherwise stated) we adopt, for the pair-component fluids, the set of dynamical equations:

$$
\begin{aligned}
\frac{\partial n_{j}}{\partial t}+\nabla \cdot\left(n_{j} \mathbf{u}_{j}\right) & =0 \\
\frac{\partial \mathbf{u}_{j}}{\partial t}+\mathbf{u}_{j} \cdot \nabla \mathbf{u}_{j} & =\frac{q_{j}}{m_{j}}\left(\mathbf{E}+\frac{1}{c} \mathbf{u}_{j} \times \mathbf{B}\right),
\end{aligned}
$$

The (total) electric and magnetic fields, $\mathbf{E}=-\nabla \phi$ and $\mathbf{B}$, obey Maxwell's laws:

$$
\begin{aligned}
& \frac{1}{c} \frac{\partial \mathbf{B}}{\partial t}=-\nabla \times \mathbf{E}, \\
& \frac{1}{c} \frac{\partial \mathbf{E}}{\partial t}=\nabla \times \mathbf{B}-\frac{4 \pi}{c} \sum_{j} n_{j} q_{j} \mathbf{u}_{j}
\end{aligned}
$$

and also obey Poisson's equation and Gauss' law(s):

$$
\nabla \cdot \mathbf{E}=-\nabla^{2} \phi=4 \pi \sum_{j=1,2,3} q_{j} n_{j}, \quad \nabla \cdot \mathbf{B}=0 .
$$


At equilibrium, overall charge neutrality is assumed. We take the direction of wave propagation together with $\mathbf{B}_{\mathbf{0}}$ to define the $x-z$ plane, by taking $\mathbf{k}=k \hat{x}$ and $\mathbf{B}_{\mathbf{0}}=B_{0, x} \hat{x}+B_{0, z} \hat{z}=B_{0}(\cos \theta \hat{x}+\sin \theta \hat{z})$; see Fig. 6 . All quantities are assumed to vary along the direction of propagation, i.e. $\nabla \rightarrow$ $\partial / \partial x$ (thus $\nabla \times \cdot=\hat{x} \times \partial \cdot / \partial x)$. Eqs. (13b) and (11) thus imply a static $x$-magnetic field component: $B_{x}=B_{x, 0}=B_{0} \cos \theta=$ cst.

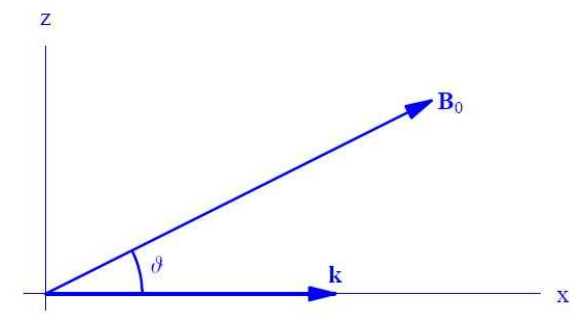

Fig. 6. The reference frame: EM wave propagation takes place along the $x$-axis, while the ambient magnetic field lies in the $x z$-plane.

The generic model described here agrees with previous studies of oblique EM wave propagation, ${ }^{11,12}$ as well as for parallel propagation. ${ }^{13}$

\section{Linear EM waves in three-component pair plasmas}

The (linear wave) dispersion relation obtained from (9)-(13) has the form

$$
D(\omega, k ; \theta)=d_{0}(\omega, k)+d_{1}(\omega, k) \sin ^{2} \theta=0,
$$

where $d_{0}$ and $d_{1}$ are polynomial expressions given by

$$
\begin{aligned}
d_{0}(\omega, k) \equiv & D(\omega, k ; \theta=0) \\
= & \left(\omega^{2}-\omega_{p, \text { eff }}^{2}\right) \\
& \times\left\{\left[\left(\omega^{2}-c^{2} k^{2}\right)\left(\omega^{2}-\Omega^{2}\right)-\omega^{2} \omega_{p, e f f}^{2}\right]^{2}-\omega^{2} \Omega^{2}\left(\omega_{p, 1}^{2}-\omega_{p, 2}^{2}\right)^{2}\right\} \\
= & \left(\omega^{2}-\omega_{p, \text { eff }}^{2}\right) \\
& \times\left\{(\omega+\Omega)\left[-\left(\omega^{2}-c^{2} k^{2}\right)(\omega-\Omega)+\omega \omega_{p, 1}^{2}\right]+\omega(\omega-\Omega) \omega_{p, 2}^{2}\right\} \\
& \times\left\{(\omega-\Omega)\left[-\left(\omega^{2}-c^{2} k^{2}\right)(\omega+\Omega)+\omega \omega_{p, 1}^{2}\right]+\omega(\omega+\Omega) \omega_{p, 2}^{2}\right\},
\end{aligned}
$$

and

$d_{1}(\omega, k ; \theta)=-c^{2} k^{2} \Omega^{2}\left\{c^{2} k^{2} \omega_{p, e f f}^{2}\left(\omega^{2}-\Omega^{2}\right)+\omega^{2}\left[4 \omega_{p, 1}^{2} \omega_{p, 2}^{2}-\left(\omega^{2}-\Omega^{2}\right) \omega_{p, e f f}^{2}\right]\right\}$.

Here we have defined the effective (total) plasma frequency $\omega_{p, e f f}=\left(\omega_{p, 1}^{2}+\right.$ $\left.\omega_{p, 2}^{2}\right)^{1 / 2}$ (cf. definitions in $\S 1$ ) and the (common among $1^{+}$and $2^{-}$) cyclotron 
frequency $\Omega=Z e B_{0} /(m c)$. Note that $d_{0}$ is a 10th order polynomial in the frequency $\omega$, while $d_{1}$ is a 4 th order polynomial in $\omega$; in both quantities, only even terms are present, so that $d_{0}\left(d_{1}\right)$ is essentially a quintic (quartic) polynomial in $\omega^{2}$. Therefore, up to 5 roots for $\omega^{2}$ may exist, hence 5 distinct propagating modes for the (real part of the) frequency $\omega(k)$ (even in $k$ ), depending on the angle $\theta$ and relevant parameter values.

Dimensionless form of the dispersion relation. The dispersion relation may be cast into a reduced form, by defining appropriate scales. Following Cramer et al, ${ }^{14}$ we define the density mismatch parameter

$$
\eta=\frac{n_{+, 0}-n_{-, 0}}{n_{+, 0}+n_{-, 0}}
$$

(see that $\omega_{p, 1}^{2}-\omega_{p, 2}^{2}=\eta \omega_{p, e f f}^{2}$ ), which measures deviation from pair-ion neutrality (the "pure" pair plasma case is recovered for $\eta \rightarrow 0$, i.e. $\omega_{p,+}=$ $\omega_{p,-}$; the case $\eta \neq 0$ thus indicates the existence of a third species, or an overall neutrality violation in the plasma composition, at equilibrium). We define the reduced wave frequency, wavenumber and plasma frequency

$$
f=\omega / \Omega, \quad \kappa=c k / \Omega, \quad h=\omega_{p, e f f}^{2} / \Omega^{2}=\left(\omega_{p, 1}^{2}+\omega_{p, 2}^{2}\right) / \Omega^{2},
$$

respectively; see that $\omega_{p, 1 / 2}^{2} / \Omega^{2} \rightarrow(1 \pm \eta) h / 2$. Eqs. $(15,16)$ thus become

$$
\begin{aligned}
\hat{d}_{0}(\omega, k) \equiv & d_{0} / \Omega^{10} \\
= & \left(f^{2}-h^{2}\right)\left\{\left[\left(f^{2}-\kappa^{2}\right)\left(f^{2}-1\right)-f^{2} h^{2}\right]^{2}-f^{2} \eta^{2} h^{2}\right\} \\
= & \left(f^{2}-h^{2}\right) \\
& \times\left\{(f+1)\left[-\left(f^{2}-\kappa^{2}\right)(f-1)+f(1+\eta) h / 2\right]+f(f-1)(1-\eta) h / 2\right\} \\
& \times\left\{(f-1)\left[-\left(f^{2}-\kappa^{2}\right)(f+1)+f(1+\eta) h / 2\right]+f(f+1)(1-\eta) h / 2\right\},
\end{aligned}
$$

and

$$
\hat{d}_{1}(\omega, k ; \theta) \equiv d_{1} / \Omega^{4}=-\kappa^{2}\left\{\kappa^{2} h\left(f^{2}-1\right)+f^{2}\left[\left(1-\eta^{2}\right) h^{2}-\left(f^{2}-1\right) h\right]\right\},
$$

Parallel EM wave propagation. For $\theta=0$, expression (14) reduces to $d_{0}=0$, implying (temporarily recovering dimensions, for clarity):

$$
\omega^{4}-\omega^{2}\left(\omega_{p, \text { eff }}^{2}+\Omega^{2}+c^{2} k^{2}\right) \mp \omega \Omega\left(\omega_{p, 1}^{2}-\omega_{p, 2}^{2}\right)+c^{2} k^{2} \Omega^{2}=0,
$$

along with trivial plasma oscillations at $\omega=\omega_{p, e f f}$. A number of distinct modes are therefore present. Note that the deviation from incompressibility (i.e. for non-zero values of $n_{1,0}-n_{2,0} \sim \omega_{p, 1}^{2}-\omega_{p, 2}^{2}$ ), due to the existence of 
the third (fixed ion) species (e.g. ions in e-p-i plasmas, or "dust" defects in pair-ion, e.g. fullerene, plasmas), leads to the appearance of extra branches (which merge back into one another in the pure p.p. limit).

The modes described by Eq. (21) have been briefly analyzed in Ref. 14 (relying on Ref. 13). This equation may be cast in the form ${ }^{14}$

$$
\left(f^{2}-1\right)\left(f^{2}-\kappa^{2}\right)-f^{2} h \pm \eta h f=0
$$

where all (dimensionless) quantities were defined above.

Interestingly, in the pure pair-plasma case (i.e. for $\eta=0),(22)$ can be solved exactly for $f^{2}$, leading (apart from $f= \pm h$ ) to

$$
f^{2}=\frac{1}{2}\left(1+\kappa^{2}+h\right)\left\{1 \pm\left[1-4 \kappa^{2} /\left(1+\kappa^{2}+h\right)^{2}\right]^{1 / 2}\right\}
$$

i.e.

$$
f^{2} \approx \frac{1}{2}\left(1+\kappa^{2}+h\right)\left\{1 \pm\left[1-2 \kappa^{2}(1-2 h)\right]\right\}
$$

for small wavenumber $\kappa$ (and, say, plasma frequency $h$ ). One thus obtains (lower branch) acoustic mode:

$$
f_{-}^{2} \approx\left(1+\kappa^{2}+h\right) \kappa^{2}(1-2 h) \approx(1-2 h) \kappa^{2}+\mathcal{O}\left(\kappa^{2}\right),
$$

and an (upper branch) optic-type mode:

$$
f_{+}^{2} \approx\left(1+\kappa^{2}+h\right)\left[1-\kappa^{2}(1-2 h)\right] .
$$

Switching back to $\eta \neq 0$, the effect of the density mismatch, which results e.g. from the existence of a third (fixed ion) species, is to split the two linearly polarized EM modes (present in p.p. ${ }^{12}$ ) to four distinct circularly polarized modes. ${ }^{14}$ Focusing on the behavior near $k=0$, one finds that three out of these modes present a frequency cutoff, i.e. $\omega(k=0) \neq 0$, below which no wave propagates. In the vicinity of $f \approx 0$, and for small $\eta$ and $h$, one finds analytically that the Alfvén type wave which occurs for $\eta=0$, splits into two modes, one of which presents a cutoff at $f_{0}=|\eta| h /(1+h)$. The behavior presented here is depicted in Fig. 7 .

Perpendicular EM wave propagation. For $\theta=\pi / 2$, expression (14) reduces to:

$$
D\left(\omega, k ; \frac{\pi}{2}\right)=d_{\perp, 1}(\omega, k) d_{\perp, 2}(\omega, k)=0,
$$

where

$$
\begin{aligned}
d_{\perp, 1}(\omega, k)= & -\omega^{6}+\omega^{4}\left[c^{2} k^{2}+2\left(\Omega^{2}+\omega_{p, \text { eff }}^{2}\right)\right] \\
& -\omega^{2}\left[\left(\Omega^{2}+\omega_{p, e f f}^{2}\right)^{2}-c^{2} k^{2}\left(2 \Omega^{2}+\omega_{p, e f f}^{2}\right)\right] \\
& +\Omega^{2}\left[c^{2} k^{2}\left(\Omega^{2}+\omega_{p, e f f}^{2}\right)+\left(\omega_{p, 1}^{2}-\omega_{p, 2}^{2}\right)^{2}\right]
\end{aligned}
$$



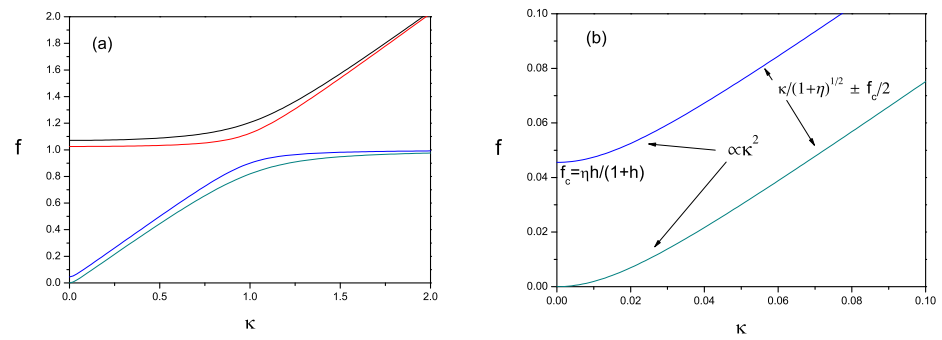

Fig. 7. Linear dispersion relation for EM waves in a three component pair-ion (or epi) plasma: the reduced frequency $f=\omega / \Omega$ is depicted against the reduced wavenumber $\kappa=c k / \Omega$; (a) full frequency range; (b) focusing near the origin. Here $\eta=0.5, h=0.1$ (definitions in the text).

and

$$
d_{\perp, 2}(\omega, k)=\omega^{2}-\omega_{p, e f f}^{2}-c^{2} k^{2}
$$

The latter equation defines the ordinary (or O-) mode; ${ }^{1}$ this is a robust perpendicular EM mode, whose dispersion characteristics do not depend on the ambient magnetic field, i.e., it bears the same form for $e-i$ plasmas and, in fact, for unmagnetized plasmas. Adopting the O-mode has enabled us to advance in analytical tractability, as regards nonlinear EM wave dynamics, ${ }^{9}$ as we shall see below. This study ${ }^{9}$ is reminiscent of an earlier study of the Omode, with respect to modulation effects, yet for unmagnetized plasmas. ${ }^{15}$

The "pure" pair-plasma limit for arbitrary $\boldsymbol{\theta}$. In the absence of the 3rd (fixed ion) species, one recovers, setting $\omega_{p, 1}=\omega_{p, 2}=\omega_{p}$ ) in (14):

$$
\begin{aligned}
\left.D\right|_{p . p .}(\omega, \theta) & =\left[\left(\omega^{2}-c^{2} k^{2}\right)\left(\omega^{2}-\Omega^{2}\right)-2 \omega^{2} \omega_{p}^{2}\right] \\
& \times\left[\omega^{2}\left(\omega^{2}-c^{2} k^{2}-2 \omega_{p}^{2}\right)\left(\omega^{2}-\Omega^{2}-2 \omega_{p}^{2}\right)-2 c^{2} k^{2} \Omega^{2} \omega_{p}^{2} \cos ^{2} \theta\right]
\end{aligned}
$$

Setting the first quantity within brackets to zero, one recovers:

$$
\omega^{4}-\left(c^{2} k^{2}+\Omega^{2}+2 \omega_{p}^{2}\right) \omega^{2}+c^{2} k^{2} \Omega^{2}=0,
$$

which coincides $^{g}$ with Eq. (9) in Ref. 12 (also see (24)-(26) in Ref. 3a. Eq. (31) represents the dispersion relation of an EM wave which propagates

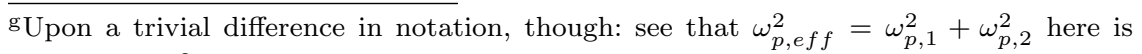
denoted by $\omega_{p}^{2}$ in Refs. 12 and 14 . 
for any value of the pitch angle $\theta$, and whose only non-vanishing electric field component $E_{y}$ is perpendicular to the plane spanned by the magnetic field $\mathbf{B}_{\mathbf{0}}$ and the wave vector $\mathbf{k}$ (i.e. $E_{x}=E_{z}=0$ ); this mode is always characterized by charge neutrality $\left(n_{i}=n_{e}=n \neq n_{0}\right.$, off equilibrium), for $\theta \neq 0$. For parallel propagation $\left(\theta=0\right.$, or $\left.B_{z}=0\right)$, this mode corresponds to a splitting of the incompressible $\left(n_{i}=n_{e}=n_{0}\right)$, circularly polarized EM waves (present in e-i plasmas) into two orthogonal, linearly polarized EM waves, both obeying Eq. (31). For perpendicular propagation $(\theta=\pi / 2$, or $\left.B_{x}=0\right)$, this mode is part of the extraordinary (X) mode; ${ }^{1}$ also see (21)(22) in Ref. 3a, and the discussion therein. See that both relations (21) merge into (31) for $\omega_{p, 1}=\omega_{p, 2}$; vice versa, in the presence of a 3rd species (e.g. in $e-p$ - $i$ plasmas), this mode splits into the 2 parts in (21).

On the other hand, upon setting the second quantity within brackets, in $r h s(30)$ to zero, one recovers exactly ${ }^{\mathrm{g}}$ Eq. (10) in Ref. 12, representing an EM mode propagating in pair plasmas, for which $E_{y}=0$ (i.e. no electric field is generated perpendicular to the plane defined by $\mathbf{B}_{\mathbf{0}}$ and $\mathbf{k}$ ). Interestingly, for $\theta=\pi / 2$, one obtains a pair of (decoupled) dispersion relations, namely $\omega^{2}=2 \omega_{p}^{2}+c^{2} k^{2}$ (corresponding to an incompressibly, linearly polarized ordinary $(\mathrm{O})$ mode, ${ }^{1}$ with $E_{z} \neq 0$ ) and $\omega^{2}=2 \omega_{p}^{2}+\Omega^{2}$ (representing a fixed frequency, pure upper-hybrid mode, with $\left.E_{x} \neq 0\right) .{ }^{12}$

The case of parallel EM wave propagation in p.p. is obtained either by setting $\theta=0$ in (30), or by setting $\omega_{p, 1}=\omega_{p, 2}=\omega_{p}$ in (15):

$\left.D_{0}\right|_{p . p .}(\omega, \theta=0)=-i \omega^{3}\left(\omega^{2}-2 \omega_{p}^{2}\right)\left[\omega^{4}-\left(c^{2} k^{2}+\Omega^{2}+2 \omega_{p}^{2}\right) \omega^{2}+c^{2} k^{2} \Omega^{2}\right]^{2}$,

thus recovering the $E_{y}$-mode discussed above, plus trivial (non-propagating, since pressure effects are neglected) plasma oscillations at $\omega=\sqrt{2} \omega_{p}$.

\section{Nonlinear EM harmonic modes}

The multiple scales technique presented in $\S 4$ above has been employed for EM modes in p.p.. The results have been presented in Ref. 9, for the ordinary (O-)mode, while part of the more general results ${ }^{16}$ have appeared (yet in a preliminary form) in Ref. 17. In the following, we shall present some essential results of the nonlinear analysis, while an interested reader is referred to the references for details on the tedious calculation.

The algebraic manipulation of the 1st order 1st harmonic $(n=1, l=1)$ amplitude evolution equations (9)-(13) provides a set of equations for the fluid densities and velocities vs. the E/M field components. Furthermore, a 2nd-order correction is obtained, for all quantities, incorporating a 2nd- 
and a zeroth-phase-harmonic $(\mathrm{s})^{\mathrm{h}}$. For the ordinary mode, the solution (up to $\sim \epsilon^{2}$ ) can be summarized as ${ }^{9}$

$$
\begin{aligned}
& n_{j}=n_{j, 0}+\epsilon c_{j}^{(11)} B_{y}^{\prime} e^{i \phi_{c}}+\epsilon^{2}\left[c_{j}^{(22)}{B^{\prime}}_{y}^{2} e^{i 2 \phi_{c}}+n_{j}^{(20)}\right] \\
& \mathbf{u}_{\mathbf{j}}=\mathbf{0}+\epsilon c_{j, z}^{(11)} B_{y}^{\prime} e^{i \phi_{c}} \hat{z}+\epsilon^{2}\left\{c_{j, z}^{(21)} \frac{\partial B_{y}^{\prime}}{\partial X_{1}} e^{i \phi_{c}} \hat{z}+{B^{\prime}}_{y}^{\prime 2} e^{i 2 \phi_{c}}\left[c_{j, x}^{(22)} \hat{x}+c_{j, y}^{(22)} \hat{y}\right]+\mathbf{u}_{\mathbf{j}}^{(20)}\right\} \\
& \mathbf{E}=\mathbf{0}+\epsilon c_{e l, z}^{(11)} B_{y}^{\prime} e^{i \phi_{c}} \hat{z}+\epsilon^{2}\left\{c_{e l, z}^{(21)} \frac{\partial B_{y}^{\prime}}{\partial X_{1}} e^{i \phi_{c}} \hat{z}+{B^{\prime}}_{y}^{2} e^{i 2 \phi_{c}}\left[c_{e l, x}^{(22)} \hat{x}+c_{e l, y}^{(22)} \hat{y}\right]+\mathbf{E}^{(20)}\right\} \\
& \mathbf{B}=B_{0} \hat{z}+\epsilon B_{y}^{\prime} e^{i \phi_{c}} \hat{y}+\epsilon^{2}\left[c_{B, y}^{(21)} \frac{\partial B^{\prime}}{\partial X_{1}} e^{i \phi_{c}} \hat{y}+c_{B, z}^{(22)}{B^{\prime}}_{y}^{2} e^{i 2 \phi_{c}} \hat{z}+\mathbf{B}^{(20)}\right] .
\end{aligned}
$$

Here $j=1,2 \equiv+,-), B_{y}^{\prime}=B_{y}^{(11)} / B_{0}$ and $\phi_{c}=k x-\omega t$. The arbitrary zeroth-order corrections satisfy

$$
u_{1, x}^{(20)}=-u_{2, x}^{(20)}=c E_{y}^{\prime(20)}, \quad u_{1, y}^{(20)}=-u_{2, y}^{(20)}=-c E_{x}^{\prime(20)} .
$$

It is worth mentioning that the compatibility conditions, imposed at orders $\epsilon^{2}$ and $\epsilon^{3}$ for secular terms to annihilate, respectively take the form

$$
\left(\frac{\partial}{\partial T_{1}}+v_{g} \frac{\partial}{\partial X_{1}}\right) \tilde{B}_{\perp}=0
$$

$\left[v_{g}\right.$ here denotes the EM wave group velocity $\omega^{\prime}(k)=-(\partial D / \partial k) /(\partial D / \partial \omega)$, as it results from the dispersion relation $D(\omega, k)=0$ - see (14) - obtained previously $\left.{ }^{\mathrm{i}}\right]$ and

$$
i \frac{\partial \tilde{B}_{\perp}}{\partial \tau}+P \frac{\partial^{2} \tilde{B}_{\perp}}{\partial \xi^{2}}+Q\left|\tilde{B}_{\perp}\right|^{2} \tilde{B}_{\perp}=0 .
$$

Here, $\tilde{B}_{\perp}=\left[B_{z}^{(11)}+C B_{y}^{(11)}\right] / B_{0}$ is a linear combination of the magnetic field components $\perp$ to the propagation direction, and $C$ is a complex quantity:

$$
C(\omega, k ; \theta)=-2 i \omega \Omega\left(\omega_{p, 1}^{2}-\omega_{p, 2}^{2}\right) \cos \theta \frac{C_{1}}{C_{2}}=0,
$$

where

$$
C_{1}=4 c^{2} k^{2}\left(\omega^{2}-\Omega^{2}\right)\left(\omega^{2}-\omega_{p, e f f}^{2}\right)+2 \Omega^{2} \omega_{p, e f f}^{2}\left(\omega^{2}-\omega_{p, e f f}^{2}-c^{2} k^{2}\right) \sin ^{2} \theta
$$

h The structure of the lengthy algebra and the resulting expressions obtained at orders $\epsilon^{1}$ and $\epsilon^{2}$ is (are) reported in Sections V and VI, respectively, of Ref. 17. Details to appear elsewhere. $^{16}$

${ }^{\mathrm{i}}$ In specific, differentiating $D(\omega(k), k)=0$ with respect to $k$ gives:

$$
\frac{\partial D}{\partial k}+\frac{\partial D}{\partial \omega} \frac{d \omega}{d k}=0, \quad \text { hence } \quad \frac{d \omega}{d k}=-\frac{\frac{\partial D}{\partial k}}{\frac{\partial D}{\partial \omega}} .
$$


and

$$
\begin{aligned}
& C_{2}=8 c^{2} k^{2}\left(\omega^{2}-\Omega^{2}\right)\left(\omega^{2}-\omega_{p, e f f}^{2}\right)\left[\omega^{2}\left(\omega^{2}-\Omega^{2}-\omega_{p, e f f}^{2}\right)-c^{2} k^{2}\left(\omega^{2}-\Omega^{2}\right)\right] \\
& +4 \Omega^{2} \omega_{p, e f f}^{2} \sin ^{2} \theta \\
& \quad \times\left[2 c^{4} k^{4}\left(\omega^{2}-\Omega^{2}\right)-i \omega \Omega\left(\omega_{p, 1}^{2}-\omega_{p, 2}^{2}\right)\left(\omega^{2}-\omega_{p, e f f}^{2}-c^{2} k^{2}\right) \cos \theta\right] .(37)
\end{aligned}
$$

Interestingly, for parallel EM wave propagation (i.e. for $\theta=0), C \rightarrow \pm i=$ $e^{ \pm i \pi / 2}$, suggesting a phase difference of $\pm \pi / 2$ among $B_{y}$ and $B_{z}$, specifically when the frequency $\omega$ obeys the (parallel EM wave) dispersion relation (15). The slowly evolving transverse magnetic field component is then $B_{z} \pm i B_{y}= \pm i\left(B_{y} \mp i B_{z}\right) \equiv \pm i B_{\perp}^{*}$. The (anticipated) circular polarization encountered for modulated EM wavepackets propagating parallel to $\mathbf{B}_{\mathbf{0}}$ (see e.g. in Ref. ${ }^{13}$ ) in multi-component plasmas is thus recovered. Also note, for rigor, that the quantity $C$ vanishes for perpendicular EM wave propagation (i.e. for $\theta=\pi / 2$ ), for $\omega_{p, 1} \neq \omega_{p, 2}$, and also in the pure pair-ion plasma case (i.e. for $\omega_{p, 1}=\omega_{p, 2}, \forall \theta$ ).

The dispersion coefficient in Eq. 35 is given by $P=d^{2} \omega(k) / 2 d k^{2}$. The nonlinearity coefficient $Q$ is a complicated function of the angle $\theta$ and of the characteristic frequencies $\omega_{p, 1 / 2}$ and $\Omega$. For the O-mode, it has the form $Q=Q_{1}+Q_{2}$, with

$$
\begin{aligned}
& Q_{1}=Q_{A} / Q_{B} \\
& Q_{A}=3 \omega_{p, e f f}^{2}\left\{-4 \omega^{2}\left(\Omega_{1}^{2} \omega_{p, 1}^{2}+Z \Omega_{2}^{2} \omega_{p, 2}^{2}\right)+\Omega_{1} \omega_{p, 1}^{2}\left[\Omega_{1} \Omega_{2}^{2}+\left(\Omega_{1}+\Omega_{2}\right) \omega_{p, 2}^{2}\right]\right. \\
& \left.+Z \Omega_{2} \omega_{p, 2}^{2}\left[\Omega_{2} \Omega_{1}^{2}+\left(\Omega_{1}+\Omega_{2}\right) \omega_{p, 1}^{2}\right]\right\} \\
& Q_{B}=\omega\left(c_{4} \omega^{4}+c_{2} \omega^{2}+c_{0}\right) \\
& c_{4}=48 \omega_{p, e f f}^{2} \\
& c_{2}=-4\left[3 \omega_{p, \text { eff }}^{4}+3 \omega_{p, \text { eff }}^{2}\left(\Omega_{1}^{2}+\Omega_{2}^{2}\right)+\omega_{p, 1}^{2} \Omega_{1}^{2}+\omega_{p, 2}^{2} \Omega_{2}^{2}\right] \\
& c_{0}=3\left(\omega_{p, 1}^{4} \Omega_{2}^{2}+\omega_{p, 2}^{4} \Omega_{1}^{2}\right)+2 \Omega_{1} \Omega_{2} \omega_{p, 1}^{2} \omega_{p, 2}^{2}+4\left(\Omega_{1}^{2}+\Omega_{2}^{2}\right) \omega_{p, 1}^{2} \omega_{p, 2}^{2}+4 \Omega_{1}^{2} \Omega_{2}^{2} \omega_{p, \text { eff }}^{2} \\
& Q_{2}=-\frac{1}{2 \omega}\left(\frac{n_{1}^{(20)}}{n_{1,0}} \omega_{p, 1}^{2}+\frac{n_{2}^{(20)}}{n_{2,0}} \omega_{p, 2}^{2}\right)
\end{aligned}
$$

( $\Omega_{1}=\Omega_{2}$, yet $\omega_{p, 1} \neq \omega_{p, 2}$, is understood here, for three component p.p.). By further assuming $\omega_{p, 1}=\omega_{p, 2}=\omega_{p}$ ("pure" p.p., no third component), we obtain the simple expressions:

$$
P=\frac{c^{2} \omega_{p}^{2}}{\omega^{3}}, \quad Q=\frac{3 \Omega^{2} \omega_{p}^{2}}{2 \omega\left(\Omega^{2}-3 \omega^{2}\right)}-\frac{1}{2 \omega} \frac{\omega_{p}^{2}}{n_{0}}\left(n_{1}^{(20)}+n_{2}^{(20)}\right) .
$$

Concluding, the important quantity to deal with, as regards EM wavepackets in pair plasmas, is $\tilde{B}_{\perp}$ (defined above), which obeys the NLSE (35). The coefficients in the latter may be used for an investigation of the 
modulation stability profile of EM waves (and associated envelope structures predicted), along the general directions set in $\S 5$.

Modulational stability profile of EM waves in pair plasmas. The coefficients of the NLSE (35) are depicted in Fig. 8, for the case of $\theta=0$, i.e. for EM wave propagation parallel to the external magnetic field. Note the forbidden frequency region (gap) near $f=1$ (i.e. near $\omega=\Omega$ ); cf. Fig. 7. We see that, for pure p.p. (for $\eta=0$, i.e. in the absence of a third species), the coefficient product $P Q$ is positive at small frequencies (i.e. for the Alfvén type $p . p$. mode lying below the cyclotron frequency $\Omega$ ), thus prescribing modulational instability and bright-type envelope excitations. However, $P Q$ becomes negative as one approaches $f=1$ from below, so high frequency waves will tend to be stable, and propagate as dark-type envelope solitons (envelope holes). A similar alternating (positive, then negative) behavior is obtained by gradually increasing $\omega$ (above $\Omega$ ).

By "switching-on" the existence of the 3rd massive background species, the product $P Q$ ( $\alpha \beta$ in Fig. 8), becomes negative for low a frequency, thus apparently stabilizing the two sub-cyclotron modes (see in Fig. 7). This is due to a shift in sign of the dispersion coefficient (right column in Fig. 8, 2nd and 3rd rows) at low $\omega$. We have seen (cf. Fig. 7) that the linearly polarized (pure) p.p. EM acoustic mode splits into two modes (one presenting a gap; see Fig. 7b) if a 3rd species is present. Both of these mode, namely a lefthand- and a right-hand-polarized one, exhibit the described behavior.

For perpendicular propagation (O-mode), the result (38) can be employed to investigate the nonlinear amplitude profile. Neglecting $n_{j}^{(20)}$, we see that the ratio $\omega / \Omega$ bears a threshold $1 / \sqrt{3}$, below (above) which dispersion is anomalous (normal) - borrowing terminology from nonlinear optics, implying bright (dark) type excitations and modulational instability (stability) of the wavepacket amplitude. A detailed investigation of the ordinary mode in pair plasmas is carried out in Ref. 9, so lengthy details (reported therein) were chosen to be omitted here, for brevity.

\section{Summary and conclusions}

We have considered the propagation of nonlinear amplitude-modulated EM wavepackets in a multi-component plasma. By adopting a multiple scales technique, we have investigated the linear oscillation profile (arising to first order) and have succeeded in showing how secondary harmonic generation, modulational instability and envelope soliton formation may occur in pair plasmas. Both ES and EM waves have been considered. 

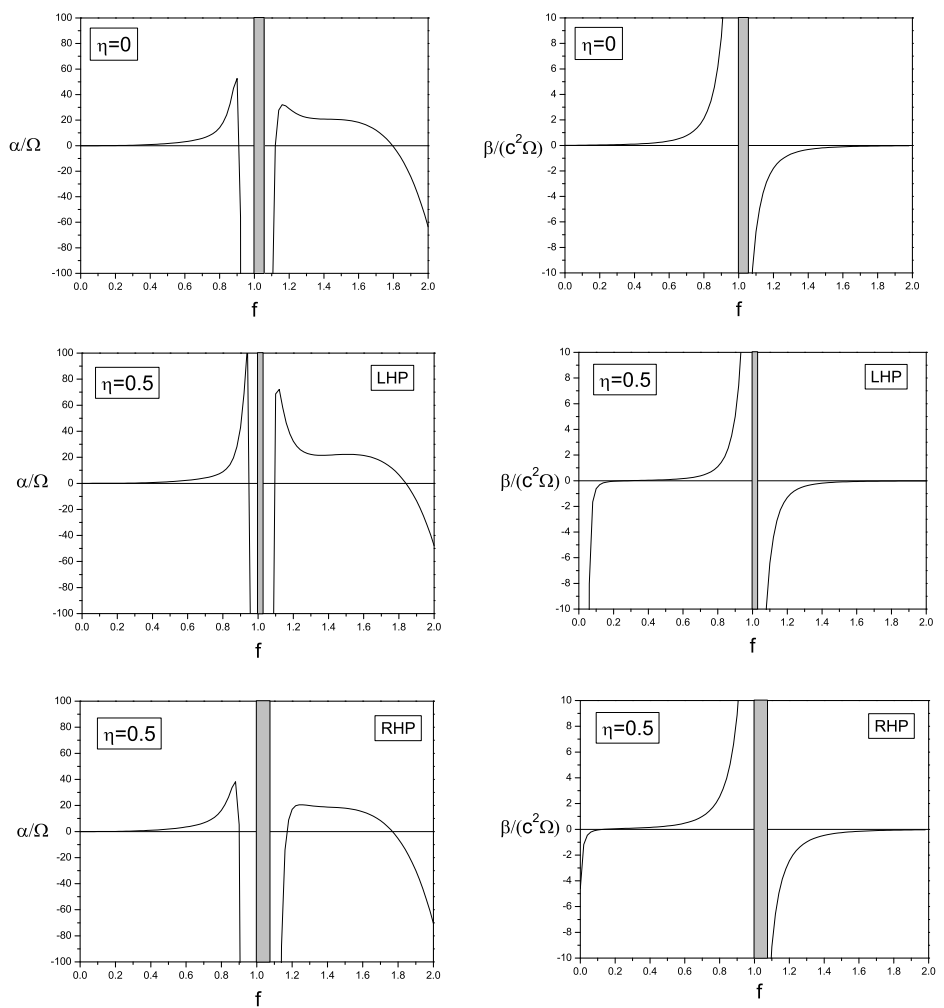

Fig. 8. The nonlinearity (left column) and dispersion (right column) coefficients in the NLS Eq. (35) for parallel EM wave propagation $(\theta=0)$ are plotted against the reduced frequency $f=\omega / \Omega$ : (a) Pure pair plasma $[\eta=0$; recall def. in (17)] - linear polarization (1st row); (b) Three-component pair plasma $(\eta=0.5)$ - left-hand polarization (2nd row); (c) Three-component pair plasma $(\eta=0.5)$ - right-hand polarization (3rd row). Note the frequency gap near $f=1$, i.e. near $\omega=\Omega$; cf. Fig. 7. From Ref. 14 .

We have shown that either a temperature difference among the two pair components or the presence of a third massive species (in "doped", say, pair-ion plasmas, or $e-p-i$ plasmas) may affect the stability profile of plasma waves. For instance, parallel EM Alfvén-like waves may be stabilized by the background component, also recovering circular polarization (lost in "pure", symmetric p.p., where the respective mode is linearly polarized).

These results are of relevance with fullerene experiments ${ }^{4}$ and $e-p-i$ plasma related observations, where they may be tested and (hopefully) 
confirmed).

\section{Acknowledgments.}

This material is based on a lecture given by I.K. in 2007 Summer College on Plasma Physics, at International Centre for Theoretical Physics (Trieste, Italy, 30 July - 24 August 2007). The Organizers of the event are warmly thanked for the invitation and the hospitality amply offered throughout his stay. The work presented here was carried out during a research visit at Universiteit Gent, Belgium in 2006 (funded by FWO, Belgian Research Fund). I.K. is grateful for that invitation, and vividly reminiscent of a fruitful stay, where multiple interactions with Prof. F. Verheest and a number of visitors have provided a warm scientific environment.

\section{References}

1. T. Stix, Waves in plasmas (New York: American Institute of Physics, 1992).

2. P. K. Shukla and A. A. Mamun, Introduction to dusty plasma physics (Institute of Physics, Bristol, 2002).

3. N. Iwamoto, Phys. Rev. E 47, 604 (1993); G.A. Stewart \& E.W. Laing, J. Plasma Phys. 47295 (1992); G. P. Zank \& R. G. Greaves, Phys. Rev. E 51, 6079 (1995).

4. W. Oohara and R. Hatakeyama, Phys. Rev. Lett. 91, 205005 (2003); W. Oohara, D. Date and R. Hatakeyama, Phys. Rev. Lett. 95, 175003 (2005).

5. I. Kourakis and P. K. Shukla, Nonlin. Proc. Geophys., 12, 407 (2005).

6. M. Remoissenet, Waves Called Solitons (Berlin, Springer-Verlag, 1996); T.Dauxois and M. Peyrard, Physics of Solitons (Cambridge Univ. Press, 2005).

7. A. Esfandyari-Kalejahi et al, J. Phys. A: Math. Gen., 39, 13817 (2006).

8. A. Esfandyari-Kalejahi et al, Phys. Plasmas, 13, 122310 (2006)

9. I. Kourakis, F. Verheest and N. Cramer, Phys. Plasmas, 14, 022306 (2007).

10. R. Fedele and H. Schamel, Eur. Phys. J. B27, 313 (2002).

11. H. Hasegawa and Y. Ohsawa, J. Phys. Soc. Japan 73(7), 1764 (2004).

12. F. Verheest and T. Cattaert, Phys. Plasmas 12, 032304 (2005).

13. S. Irie and Y. Ohsawa, J. Phys. Soc. Japan 70(6), 1585 (2001).

14. N.F. Cramer et al, Proc. 28th ICPIG (Prague, 2007), paper 1P04-04.

15. G S Lakhina \& B Buti, Astrophys. Space Sci. 79, 25 (1981).

16. I. Kourakis \& F. Verheest, unpublished material.

17. International Workshop on Frontiers of Plasma Science (2006), Abdus Salam ICTP (activity smr 1765), Trieste, Italy; online Lecture Notes, available at: http://cdsagenda5.ictp.trieste.it/full_display .php?ida=a05217. 